\title{
Establishing reliable good initial quantum efficiency and in-situ laser cleaning for the copper cathodes in the RF gun
}

\author{
F. Zhou, J. C. Sheppard, T. Vecchione, E. Jongewaard, \\ A. Brachmann, J. Corbett, S. Gilevich, and S. Weathersby \\ SLAC National Accelerator Laboratory, 2575 Sand Hill Road, Menlo Park, CA 94025, USA
}

\begin{abstract}
Establishing good initial quantum efficiency (QE) and reliable in-situ cleaning for copper cathode in the RF gun is of critical importance for the RF gun operations. Recent studies at the SLAC RF gun test bed indicated that the pre-cleaning (plasma cleaning) in the test chamber followed by copper cathode exposure to air for cathode change leads to a very low initial QE in the RF gun, and also demonstrated that without the pre-cleaning good initial $\mathrm{QE}>4 \times 10^{-5}$ can be routinely achieved in the RF gun with the cathodes of $\mathrm{QE}<1 \times 10^{-7}$ measured in the test chamber. QE can decay over the time in the RF gun. The in-situ laser cleaning technique for copper cathodes in the RF gun are established and refined in comparison to previous cleaning at the linac coherent light source, resulting in an improved QE and emittance evolutions. The physics of the laser cleaning process is discussed. It is believed that the reflectivity change is one of the major factors for the QE boost with the laser cleaning.
\end{abstract}

Keywords: photocathode; laser cleaning; quantum efficiency; emittance

\section{Introduction}

The linac coherent light source (LCLS) located at the SLAC National Accelerator Laboratory has been successfully operated for users for more than 5 years. Its copper-cathode photo-injector has produced an ultra-low emitance and ultra-fast electron beam for the x-ray free electron laser (XFEL). Establishing good initial quantum efficiency (QE) $>4 \times 10^{-5}$ and in-situ cleaning for the cathodes in the radio frequency (RF) gun is crucial for LCLS injector system operations. Three identical copper cathodes have been changed for the LCLS operations but with very different initial $\mathrm{QE}$ values in between $5 \times 10^{-6}$ and $5 \times 10^{-5}$ in the gun. The lower $\mathrm{QE}\left(<1 \times 10^{-5}\right)$ makes the LCLS drive laser system operations difficulty in term of pulse energy and beam profile stability. At high laser power densities, the optical components are readily deteriorated resulting in deformation of the laser profile on the cathode, thereby impacting electron beam quality. It is therefore needed to establish reliable good initial $\mathrm{QE}>4 \times 10^{-5}$ in the LCLS RF gun through understanding the cause of the non-reproducible QE. In addition, Even with high initial QE, over time the QE can decay so that the in-situ cleaning of the cathode surface in the RF gun is also needed for efficient operations.

This paper addresses both needs: to establish good initial QE in the gun and develop gun in-situ cleaning of cathode surface to maintain good QE during operations. In Section 2, we discuss the cause of very low initial QE for photocathodes previously installed in the LCLS gun and then present good initial QE established at the SLAC's Accelerator Structure Test Area (ASTA) with RF processing. Section 3 describes systematic studies leading to an improved laser cleaning procedure, which results in better electron beam performance than the previous one at the LCLS gun [1]. Section 3 also presents pointing stability requirements for the UV laser at the cathode surface during the laser cleaning process and discusses the physics of the laser cleaning. Lastly, the results of this work are summarized. 


\section{Establishment of good initial quantum efficiency in the RF gun}

An RF gun test bed located at the ASTA has been constructed and commissioned [2] to study the photocathodes for the support of LCLS injector cathode operations. The beamline of the ASTA gun test bed duplicates the existing LCLS injector gun system [3], consisting of a s-band RF gun to obtain about 5.5 MeV kinetic energy, a chirp-pulse-amplifier laser tripled to $253 \mathrm{~nm}$ wavelength, LCLS-type RF gun, a solenoid for emittance compensation, one pair of magnet correctors, a Faraday cup to measure the bunch charge, and a YAG screen to measure beam size and intrinsic emittance. Similar to the LCLS injector, the drive laser is configured for normal incident injection to the photocathode surface using a $45^{\circ}$ in-vacuum mirror.

Like worldwide most metal cathode RF gun systems, the LCLS and ASTA copper cathodes have to be exposed to the air for the cathode change due to lack of loadlock systems. The load lock systems may make systems complicated and also generate unwanted dark current with the RF contact between the cathode and cavity body. In addition, the metal cathodes (e.g., copper and $\mathrm{Mg}$ ) are relatively less sensitive to the air-exposure than semiconductor cathodes for the cathode change. So the loadlock systems are not implemented in the LCLS/ASTA RF gun systems.

\subsection{The cause for low initial QE in the LCLS RF gun}

As previously mentioned, the initial QE for the three copper cathodes installed in the LCLS gun was not

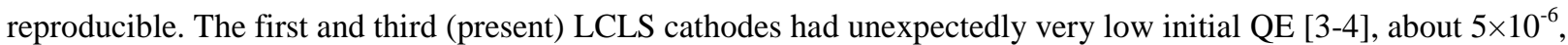
while the second one had $6.5 \times 10^{-5}$ of initial QE as expected, as illustrated in Fig.1. Lately, it was realized that both the first and third LCLS cathodes were exposed to the pre-cleaning (plasma cleaning) in the test chamber prior to installation in the RF gun, while the second one did not have the plasma cleaning process. Table 1 lists the standard steps to prepare for a cathode before installation in the LCLS RF gun including the option step 8 for the plasma cleaning. Very low QE measured in the test chamber drives to proceed to step 8, plasma cleaning in the test chamber. In the last step, the cathodes have to be exposed to air for about 3 minutes for the cathode installation in the gun due to the lack of loadlock system.

The recent experimental observations at the ASTA RF gun reveal that the laser-cleaned areas are much more susceptible to the air exposure than the non-cleaned areas. For these tests the ASTA RF gun is vented to nitrogen and then exposed to air for about 3 minutes to mimic the LCLS cathode change before its vacuum starts to be pumped down. Fig.2 (left) and (right) shows the QE maps before and after the RF gun vacuum venting to air, respectively. Before the RF gun vacuum venting, areas A, B, C, D, E, F, G and $\mathrm{H}$ on the cathode have been cleaned by the intensive laser, while the circled center area is not exposed to any laser cleaning. In Fig. 2 (left), bunch charge from areas $\mathrm{A}, \mathrm{B}$ and $\mathrm{C}$ have been evolved to about 7500 units for a given laser energy, equivalent to $1 \times 10^{-4}$ of $\mathrm{QE}$, while the cathode center area has about 3500 units of the bunch charge for the same laser energy, equivalent to about $4 \times 10^{-5}$ of QE. After gun vacuum venting, bunch charge productions from the previously cleaned areas A, B, C, D, E,

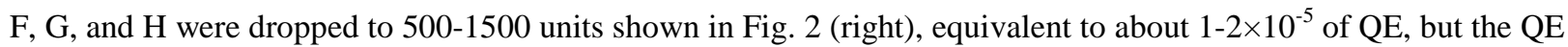
of the cathode center area still remains unchanged, at $4 \times 10^{-5}$. The experiment demonstrates that the cleaned or activated surface is susceptible to the air-exposure, indicating the previous pre-cleaning in the test chamber is the cause for the very low initial QE measured in the RF gun.

During the ASTA experiments a total of four identical cathodes are tested with no plasma cleaning prior to the installation in the ASTA RF gun. As illustrated in Fig. 1, all four ASTA cathodes have good initial QE in the RF gun ranging from $4 \times 10^{-5}$ to $8 \times 10^{-5}$. We conclude that the plasma cleaning process in the test chamber activates the 
cathode surface, making it more susceptible to the subsequent contaminations, e.g., air-exposure for cathode change. With removal of step 8 in Table 1, high initial QE can be routinely achieved in the RF gun. Note all QE measurements in the RF gun are under nominal LCLS gun operating conditions: $30^{\circ}$ of RF gun phase from zerocrossing and about $110 \mathrm{MV} / \mathrm{m}$ of peak accelerating gradient on the cathode.

Table 1. LCLS cathode preparation steps.

\begin{tabular}{|l|}
\hline Step 1: Cathode braze, dry $\mathrm{H} 2$ at $1040^{\circ} \mathrm{C}$ \\
\hline Step 2: Vacuum fire at $650^{\circ} \mathrm{C}$ for 24 hours \\
\hline Step 3: Rough machining \\
\hline Step 4: Diamond fly cut \\
\hline Step 5: Vacuum fire at $650^{\circ} \mathrm{C}$ for 24 hours \\
\hline Step 6: Weld the cathode to flange \\
\hline Step 7: Measure QE in the test chamber \\
\hline Step 8: Plasma cleaning in the test chamber (option) \\
\hline $\begin{array}{l}\text { Step 9: Exposed to air (due to lack of load lock } \\
\text { system) for the installation in the gun }\end{array}$ \\
\hline
\end{tabular}

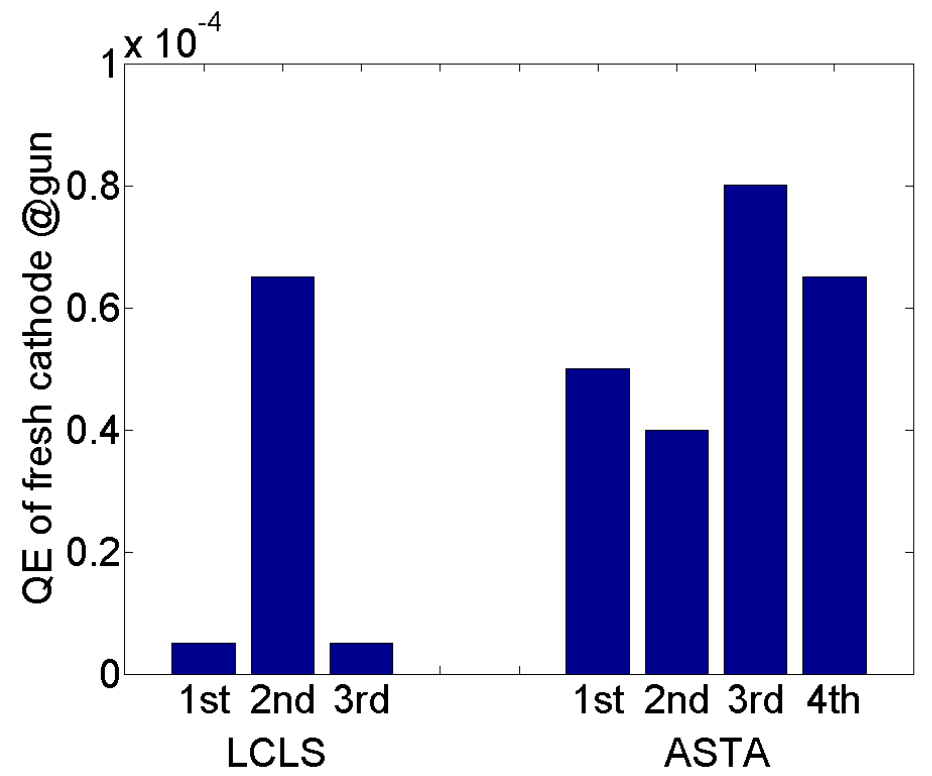

Fig.1. Initial QE of cathodes used in the LCLS and ASTA RF guns. The $2^{\text {nd }}$ LCLS cathode and all 4 ASTA cathodes did not have pre-cleaning (plasma cleaning) prior to installation in the guns, while the $1^{\text {st }}$ and $3^{\text {rd }}$ LCLS cathodes were processed by the plasma cleaning in the test chamber. 

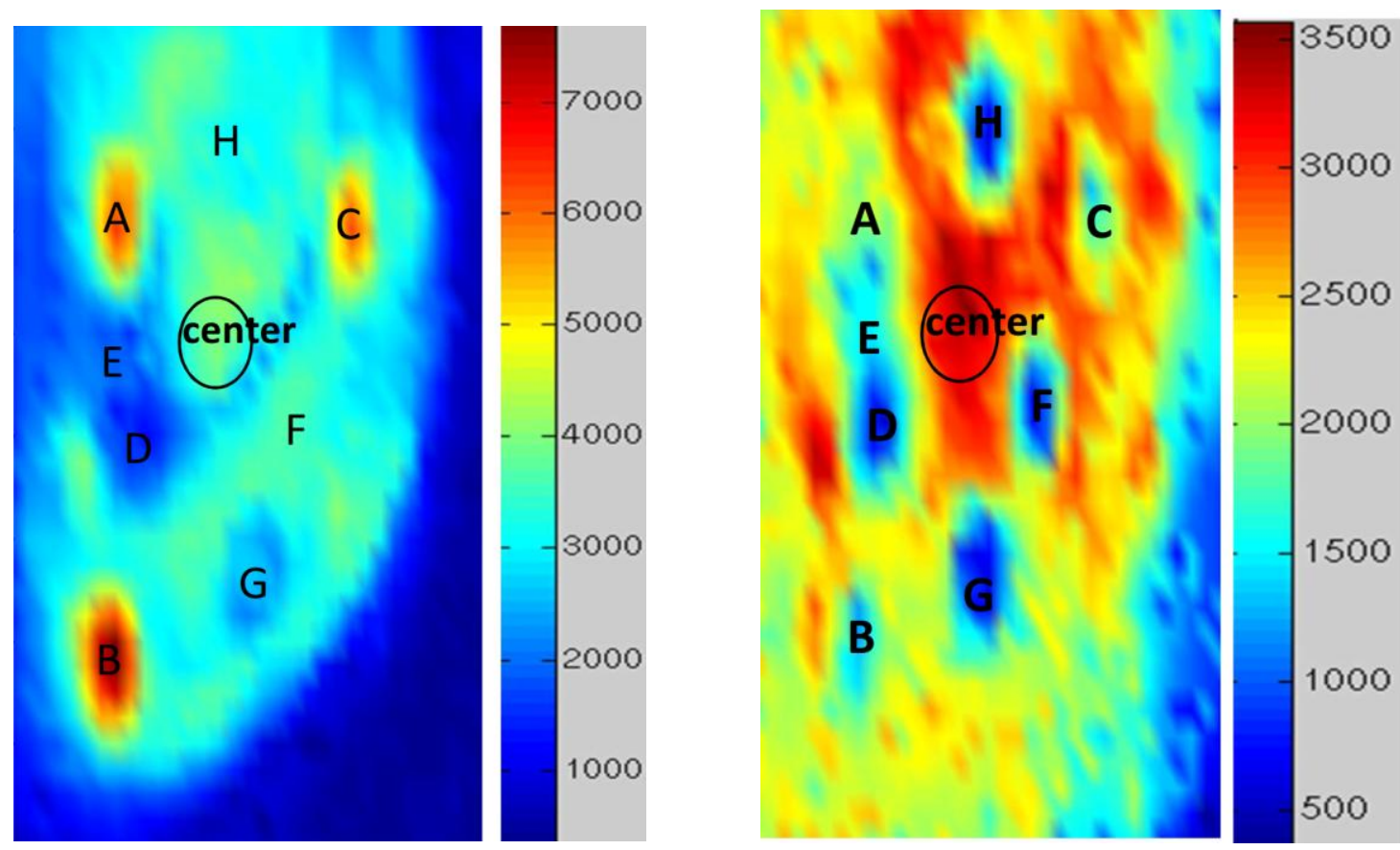

Fig.2. QE maps before (left) and after (right) the gun vacuum venting. Before the gun venting, areas A to $\mathrm{H}$ are processed by laser cleaning, while the center area is not cleaned by the intensive laser. Scale to right of each plot indicated units of the bunch charge for the same laser pulse energy. The full scales for the two plots are same 11.2 $\mathrm{mm}(\mathrm{x}) \times 8 \mathrm{~mm}(\mathrm{y})$.

\subsection{QE impact from the high-power RF processing}

For LCLS operations all three photocathodes were characterized in a test chamber prior to the installation in the RF gun. The test chamber utilizes a broadband UV light source followed by a narrow-band monochromator to select the desired photon energy. The photoemission from the cathode under $2.6 \mathrm{kV} / \mathrm{m}$ of electric field is measured with nanoammeter. Fig.3 shows one example of QE of cathode vs. photon energy in the test chamber. The QE measured in the test chamber is typically on the order of $1 \times 10^{-7}$ at the desired photon energy of $4.91 \mathrm{eV}(253 \mathrm{~nm})$. The low QE cathodes were then transferred from the test chamber directly to ASTA RF gun. The RF processing may boost QE [3] but it is never quantified how much QE boost could be. Surprisingly, following high-power RF processing all four ASTA cathodes in the gun have demonstrated an initial QE $>4 \times 10^{-5}$, two orders of magnitude higher than in the test chamber. The calculated QE enhancement from the Schottky effect in the RF gun, the ratio of QE including Schottky effect to the one without including Schottky effect, is less than an order of magnitude, as shown in Fig. 4, for a copper work function between 4.3-4.7 eV. The two orders of magnitude of QE enhancement in the RF gun therefore cannot be explained by the Schottky effect alone. It is noticed that the high-power RF operation boosts QE at the ASTA RF gun, as shown in Fig. 5. For a new cathode installed in the ASTA RF gun, the initial QE is about $3 \times 10^{-5}$ for the first day of electron beam operation. Then the QE increases by $50 \%$ after a few days of RF operations. It is logical to assume the RF processing conditions the cathode by removing surface contamination. In addition, the measured reflectivity of the cathode surface is changed from about $30 \%$ in the test chamber prior to installation in the gun to about $10 \%$ after RF processing at the desired $253 \mathrm{~nm}$ of wavelength. In combination with the Schottky effect, a much higher $\mathrm{QE}>4 \times 10^{-5}$ is observed relative to very low $\mathrm{QE}<1 \times 10^{-7}$ measured in a test chamber. 
The observations are important for understanding and developing cathode preparation procedures. Even with very low $\mathrm{QE}$ measured in the test chamber $\left(<1 \times 10^{-7}\right)$, no extra pre-cleaning in the test chamber is needed prior to installation in the gun. It is found the high-power RF processing in the gun boosts the QE to mid- $10^{-5}$ with the Schottky QE enhancement effect from a very low $\mathrm{QE}<1 \times 10^{-7}$ in the test chamber.

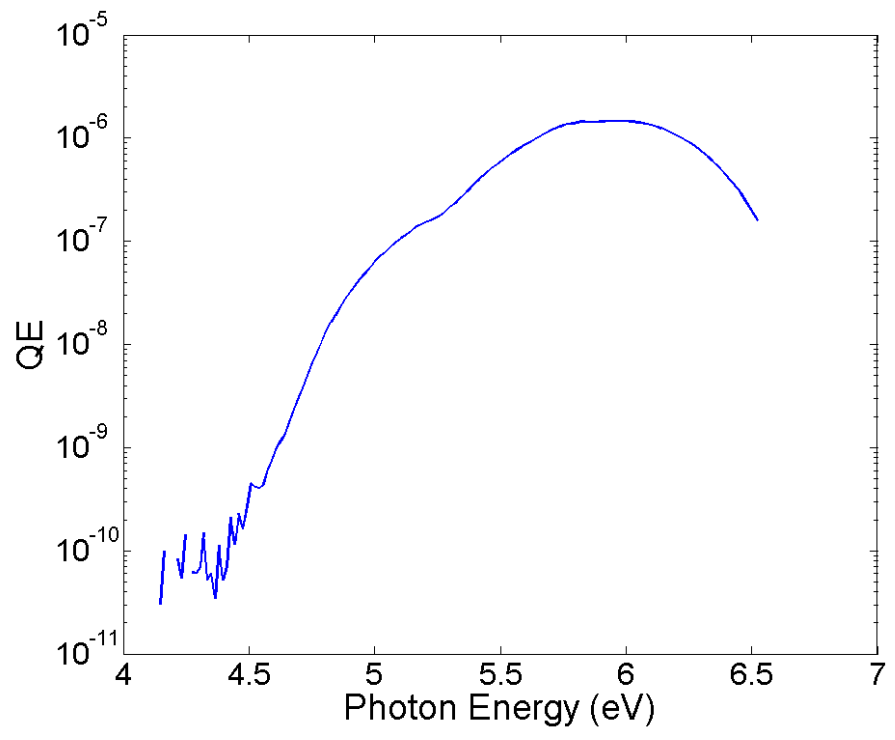

Fig.3. Typical QE measured in the test chamber prior to installation in the gun. QE is $<1 \times 10^{-7}$ at the photon energy of $4.91 \mathrm{eV}(253 \mathrm{~nm})$.

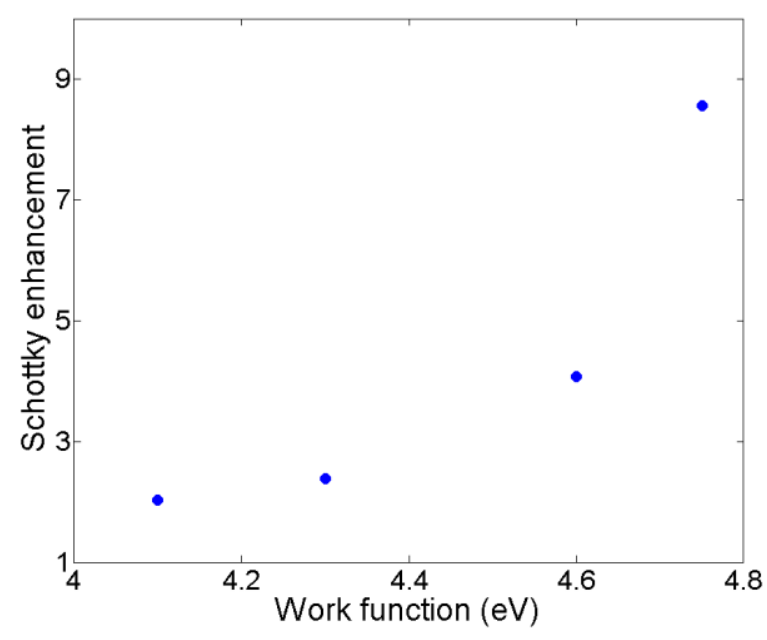

Fig.4. Calculation of QE enhancement due to the Schottky effect for a copper photocathode with work function varying between 4.3 and $4.7 \mathrm{eV}$. Schottky enhancement $=\mathrm{QE}$ (including schottky effect) $/ \mathrm{QE}$ (without schottky effect). 

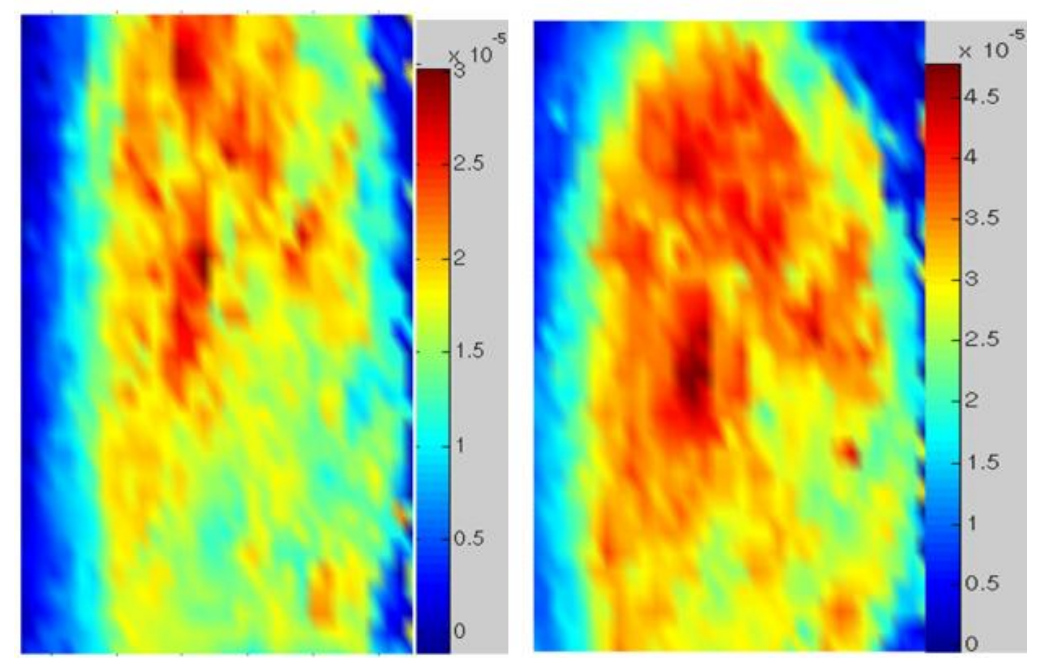

Fig.5. QE map for a new cathode in the ASTA RF gun: $3 \times 10^{-5}$ of QE for the first day of electron beam turn-on (left), $4.5 \times 10^{-5}$ one week later (right). The QE is increased by $50 \%$ after a week of RF operation. The full scales for the left and right plots are $11.2 \mathrm{~mm}(\mathrm{x}) \times 12 \mathrm{~mm}(\mathrm{y})$ and $14.1 \mathrm{~mm}(\mathrm{x}) \times 11 \mathrm{~mm}(\mathrm{y})$ respectively.

\section{In-situ laser cleaning developments at the ASTA RF gun}

Laser-based cleaning techniques have been widely used to clean metal photocathodes, such as copper and $\mathrm{Mg}$, for more than two decades [5-6]. A high-intensity laser beam, interacting with the metal cathodes, may ablate the cathode surface, removing surface contamination and possibly changing the cathode reflectivity, thereby resulting in a QE increase. However, the laser cleaning may change QE uniformity across the cathode surface, thereby changing electron beam emittance, and also generate unwanted dark current or even deteriorate cathode's crystal quality, if the laser cleaning process is too aggressive.

The previous laser cleaning was performed for the present LCLS cathode in July 2011 [1]. The QE was evolved to $1 \times 10^{-4}$ from $3 \times 10^{-5}$ over a few months following the laser cleaning. Since then, a QE value of $1 \times 10^{-4}$ is essentially unchanged for more than three years to date for $24 / 7$ users operation. The emittance was recovered to the normal value within three weeks following the laser cleaning. Although the previous LCLS laser cleaning was successful, two major concerns still remained. One concern was the reproducibility of the laser cleaning for different spots on the same cathode and for different cathodes. The other was the need to reduce emittance-recovery time and QEevolution time following the laser cleaning with refinements of the laser cleaning process. These concerns are particularly important for the LCLS, leading to further laser cleaning development at the ASTA.

\subsection{Improvements of laser cleaning at the ASTA RF gun}

The laser energy for cleaning of the current LCLS cathode performed in 2011 was fully determined by the vacuum activity in the RF gun [1]. In that case, the laser energy was set to a value for each round of the laser cleaning in order that about $0.5 \times 10^{-10}$ Torr of the RF gun vacuum rise can be observed. It took 2-3 rounds of laser cleaning to increase $\mathrm{QE}$ to the desired value, $(3-5) \times 10^{-5}$. For a better laser cleaning process, the laser energy should be set to a value with which QE is increased but with minimum gun vacuum rise. In this case, the copper crystal quality and cathode uniformity may not be affected. Ideally any minor surface change by the laser cleaning may cause a vacuum change. But in practice, observation of the RF gun vacuum rise depends on the resolution of the RF 
gun vacuum gauge and its controls, and the distance of the vacuum gauge to the cathode. To consider these practical factors, at ASTA we start the laser cleaning with a laser-energy below the observable small gun vacuum activity. For example, if a small RF gun vacuum activity of $0.5 \times 10^{-10}$ Torr is observed with $8 \mu \mathrm{J}$ of laser energy in about 40 $\mu \mathrm{m}$ rms of spot size, the actual laser energy for the first round is set to 6-7 $\mu \mathrm{J}$. Using this technique it was found that the QE could be reliably enhanced with only small amounts of vacuum activity.

Laser energy for subsequent rounds is increased in small, $0.5-1 \mu \mathrm{J}$ steps. Typical RF gun vacuum activity and laser energy for each round are shown in Fig. 6. The vacuum activity during the laser cleaning for each round at ASTA is much less than at the LCLS [1]. The laser cleaning raster step size is $30 \mu \mathrm{m}$ for a $40 \mu \mathrm{m} \mathrm{rms}$ focused laser beam size. For three of the four cathodes installed in the ASTA gun, about ten $1.2 \mathrm{~mm} \times 1.2 \mathrm{~mm}$ areas on each cathode were processed by the laser cleaning. In this case $\mathrm{QE}$ increased to (3-5) $\times 10^{-5}$ for all areas after a few rounds of laser cleaning. The $\mathrm{QE}$ and emittance evolution following the laser cleaning were consistent and reproducible for different areas and cathodes. Table 2 lists the standard laser cleaning parameters developed at ASTA, in comparison to the previous laser cleaning recipe for the LCLS cathode. The laser power density for a single laser shot at ASTA is less than at the LCLS. The number of laser cleaning shots at ASTA for each spot is also only half the number at the LCLS. The integrated laser fluence for cleaning at ASTA is therefore much less than the previously used at the LCLS. The ASTA cleaning process results in better QE evolution and faster emittance recovery, following the laser cleaning than at the LCLS. The detailed comparison of QE and emittance evolution for the laser cleaning in the LCLS and ASTA is described in Section 3.2.
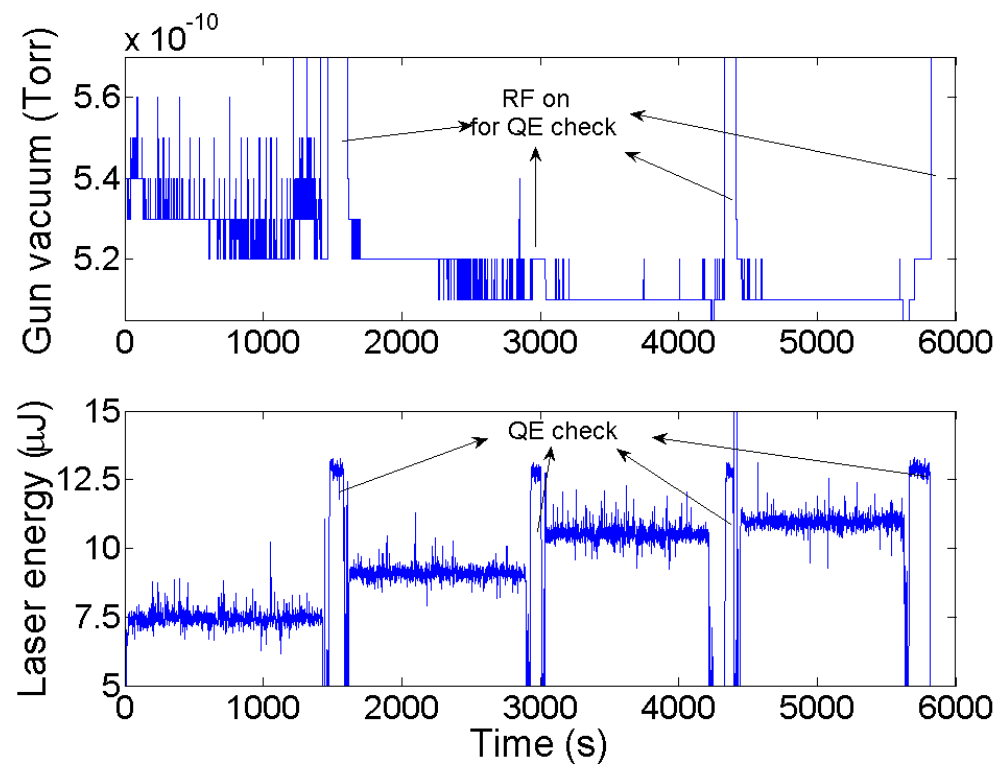

Fig.6. Laser energy and gun vacuum activity during the laser cleanings at ASTA. The QE is checked between each round of laser cleaning. 
Table 2. Laser cleaning parameters for ASTA compared to previous cleaning at the LCLS. The values in parenthesis were used at the LCLS.

\begin{tabular}{|l|l|}
\hline Laser cleaning parameters & ASTA (LCLS) \\
\hline Laser pulse length in ps FWHM & $1.6(\sim 3)$ \\
Laser size for cleaning in $\mathrm{x} / \mathrm{y}$ in $\mu \mathrm{m}, \mathrm{rms}$ & $\sim 40(\sim 30)$ \\
Laser shots per spot & $60(120)$ \\
Laser wavelength in $\mathrm{nm}$ & $253(253)$ \\
Laser raster step size in $\mathrm{x} / \mathrm{y}$ in $\mu \mathrm{m}$ & $30(30)$ \\
\hline Base gun vacuum range with RF power off & $4 \times 10^{-10}$ to $1.2 \times 10^{-9}$ Torr $\left(4 \times 10^{-10}\right.$ to $7 \times 10^{-10}$ Torr $)$ \\
\hline RF power is off during laser cleaning \\
\hline Multi-round laser cleaning: \\
- start with $7 \mu \mathrm{J}$ (17 $\mu \mathrm{J})$ \\
- laser energy for subsequent rounds is increased in $0.5-1 \mu \mathrm{J}(1-2 \mu \mathrm{J})$ \\
- take 3-8 rounds (2-3 rounds) until QE up to 3-5 $\times 10^{-5}$ \\
\hline
\end{tabular}

\subsection{QE and emittance evolution following the laser cleaning, and dark current}

Using the standard ASTA laser cleaning process, the QE can be reliably increased to $(3-5) \times 10^{-5}$ from $5 \times 10^{-6}$ $2 \times 10^{-5}$ after 3-8 rounds of the gentle cleaning. Following the laser cleaning, the QE gradually increases during either idle conditions or normal high-power RF operations. The QE eventually evolved to $>1 \times 10^{-4}$ over $2-3$ weeks, as illustrated in Fig. 7, much shorter than 4-5 months for previous LCLS cleaning. Fig. 7 shows all five areas A-E cleaned by the intense laser have similar QE evolution over time, while the QE of the center area, which was not exposed to the laser cleaning, was kept unchanged over time as expected. Note that the five areas A, B, C, D and E are cleaned on different dates, on $10^{\text {th }}, 18^{\text {th }}, 25^{\text {th }}, 32^{\text {nd }}$ and $32^{\text {nd }}$ respectively, as illustrated in the plot.

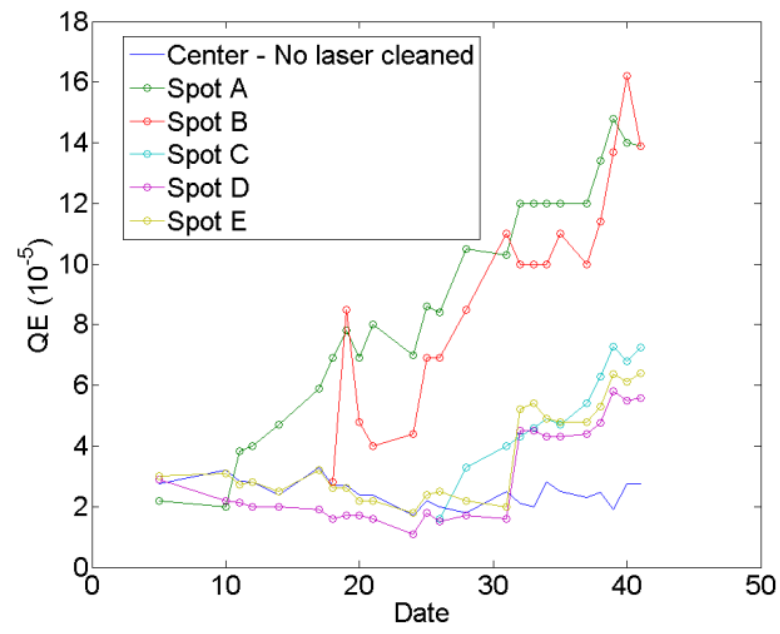

Fig.7. QE evolution for the areas with and without laser cleaning. Areas A, B, C, D and E are exposed to the laser cleaning, while the center area is not cleaned by the intensive laser. 
At ASTA, the beam energy is determined by measuring the beam rotation angle through the solenoid, and the beam size is measured at a YAG screen downstream of the solenoid. The measured beam energy is $5.5 \mathrm{MeV}$ with which the beam is space charge dominated. Thus, the intrinsic emittance is measured with very low charge 1-2 pC using the solenoid scan. Fig. 8 shows the measured intrinsic emittance before the cleaning, immediately after cleaning, and the next day after the laser cleaning. For the emittance measurements, the laser spot size on the cathode is $1 \mathrm{~mm}$ with about $3 \mathrm{~mm}$ offset in x-plane from the cathode center. The intrinsic emittance is increased immediately following laser cleaning but the next day the emittance is recovered to the value before cleaning. Highpower RF operation helps to smoothen out the surface's non-uniformity thereby improving the emittance. The emittance recovery time at ASTA is only 1-2 days, much shorter than 2-3 weeks for the previous cleaning at the LCLS. The reduced laser fluence for the cleaning at ASTA resulted in a better QE and emittance evolution than the previous laser cleaning at the LCLS.
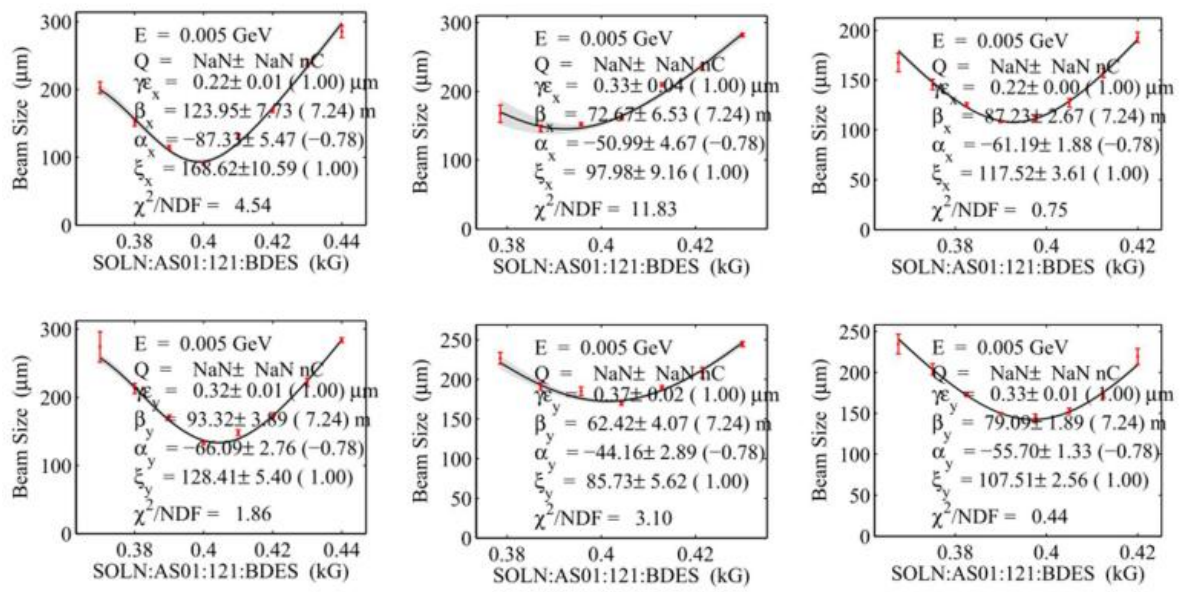

Fig.8. Measured intrinsic emittance before the laser cleaning (left), immediately after the laser cleaning (middle) and the next day after the laser cleaning (right).

Dark current is measured with a Faraday cup, located at about $1.5 \mathrm{~m}$ from the cathode. Prior to the laser cleaning the dark current from the ASTA cathode and gun is lower, $<100 \mathrm{pC}$. With the standard gentle laser cleaning, no dark current increase is observed at ASTA. However, $\sim 0.5 \mathrm{nC}$ of dark current is observed when the laser energy for the laser cleaning is intentionally doubled.

\subsection{Laser pointing requirement for the laser cleaning}

Reasonable good laser pointing stability on the cathode is required for the application of laser cleaning technique to the cathode cleaning. The focused laser size for the laser cleaning of the ASTA cathodes was about $40 \mu \mathrm{m}$ rms. According to the standard ASTA laser cleaning procedures, QE starts to increase when the laser energy is increased to $7 \mu \mathrm{J}$ with continuous 60 shots for each spot. However, on one test date the QE did not change at all after laser cleaning, even when the laser energy was increased to $28 \mu \mathrm{J}$ with nominal 60 shots for each spot on the cathode. Eventually QE was increased through doubling the number of laser shots for each spot. Later, it was realized the laser pointing stability on the cathode was bad during the laser cleaning, about $10 \mu \mathrm{m}$ rms or double the normal pointing jitter, as shown in Fig. 9. The bad laser pointing caused the failure of the laser cleaning. From this it became clear that melting the cathode surface needs a certain amount of laser shots at each spot. Unstable laser pointing significantly reduces the actual laser shots for the same spot. The laser cleaning worked again with the 
nominal laser shots, when the laser pointing stability was recovered to the normal value. According to the observations, laser pointing stability $\Delta \sigma_{x, y} / \sigma_{x, y}$ is required better than $10 \%$ to make laser cleaning work. This laser pointing requirement can be met readily for laser user facilities.

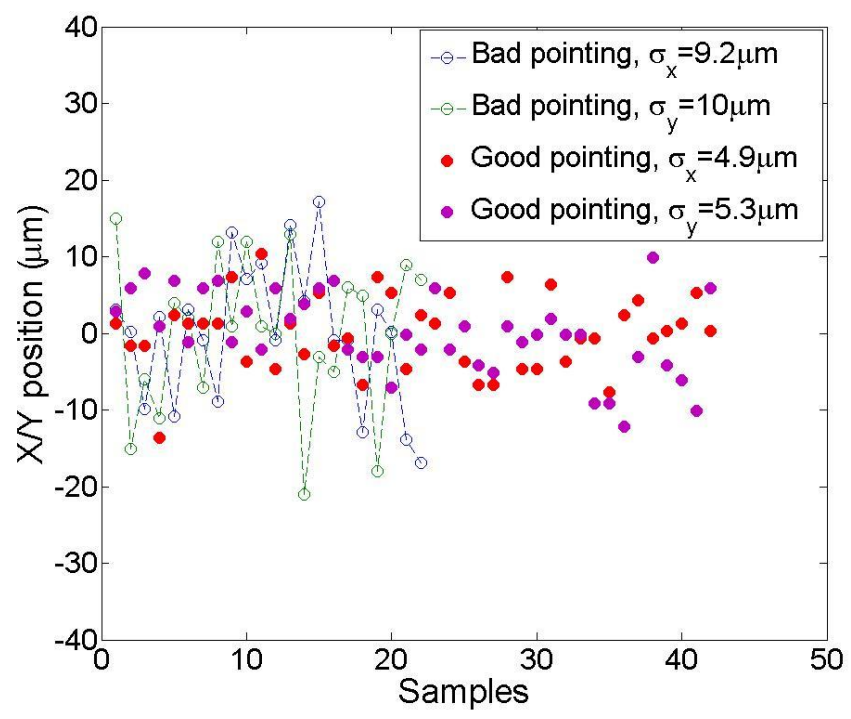

Fig.9. Laser pointing stabilities during the laser cleaning. Laser cleaning fails with the bad laser pointing.

\subsection{Discussions of physics of the laser cleaning process}

Hydrogen cleaning [7] is known as a pure chemical process through reaction of the hydrogen with surface contaminations. The chemical process may reduce the work function thereby increase the photoemission. In the past, laser cleaning had been considered as a chemical process similar to hydrogen cleaning, removing surface contaminations thereby reducing the work function. Therefore, in that picture, laser cleaning may increase the intrincis emittance with the reduced work function and the surface reflectivity is unchanged. It is noted that analytical expressions relating QE to work function $\phi_{\mathrm{w}}$ and intrinsic emittance $\varepsilon_{\mathrm{x}}$ can be given by the following equations [8]:

$$
\begin{gathered}
Q E(\lambda)=\frac{1-R(\lambda)}{1+\frac{\lambda_{\text {opt }}(\lambda)}{\lambda_{e-e}(\lambda)} \frac{\left(h v-\phi_{e f f}\right)^{2}}{8 \phi_{e f f}\left(E_{F}+\phi_{e f f}\right)}} \\
\frac{\varepsilon_{n}}{\sigma_{x}}=\sqrt{\frac{h v-\phi_{e f f}}{3 m c^{2}}}
\end{gathered}
$$

with $\phi_{e f f}=\phi_{w}-0.037947 \sqrt{E_{0}}$, where:

- $\mathrm{QE}(\lambda)$ is the $\mathrm{QE}$ dependence on the laser wavelength; $\lambda$ is the laser wavelength

- $\quad R$ is the cathode optical reflectivity, 0.43 for a copper at $253 \mathrm{~nm}$.

- $h v$ is the photon energy, about $4.91 \mathrm{eV}$ for $253 \mathrm{~nm}$ of wavelength 
- $\quad \lambda_{\text {opt }}$ is the photon absorption length of 116 angstroms

- $\quad \lambda_{\text {e-e }}$ is electron-electron mean-free path about 22 angstroms

- $\quad \mathrm{E}_{\mathrm{f}}$ is the Fermi energy, about $7 \mathrm{eV}$

- $\quad \phi_{\text {eff }}$ is the effective work function

- $\quad \mathrm{mc}^{2}$ is the rest mass energy of electron

- $\quad E_{0}$ is the applied RF electric field on the cathode, expressed by $E_{\text {peak }} \bullet \sin (\varphi)$ in units of $M V / m$

- $\quad \mathrm{E}_{\text {peak }}$ is gun's peak accelerating gradient, $110 \mathrm{MV} / \mathrm{m}$

- $\varphi$ is the gun phase from zero-crossing, $15^{\circ}$ for intrinsic emittance measurement.

For the LCLS RF gun, if the QE boost doubling from $6 \times 10^{-5}$ to $1.2 \times 10^{-4}$ were attributed to the work function change, Eqs. 1 and 2 would indicate the intrinsic emittance should increase by about $18 \%$ from typical measured value about $0.9 \mu \mathrm{m} / \mathrm{mm}$-rms to $1.06 \mu \mathrm{m} / \mathrm{mm}$-rms. However, as shown in Fig. 10, no noticeable change of intrinsic emittance was observed at the LCLS when the QE was increased by a factor of 2 following laser cleaning. In addition, the projected emittance for $150 \mathrm{pC}$ with $5 \times 10^{-6}$ of QE is very similar to the one with $1 \times 10^{-4}$. These results show the laser cleaning process does not change emittance, which indicates the work function may not change. The images of LCLS [1] and ASTA cathodes using white light, as illustrated in Fig. 11, suggest that the reflectivity for the laser cleaned areas is decreased after the laser cleaning. The two squares subject to the laser cleaning have lower visible light reflectivity than other areas without the laser cleaning. Subsequent SEM analyses shown in Fig. 12 clearly show the surface has physical change due to the laser cleaning process. Unlike hydrogen cleaning, the laser cleaning process is believed to reduce reflectivity at the cathode surface, rather than removing chemical impurities [3]. The change of reflectivity is one of the major factors for the observed QE enhancement following the laser cleaning.

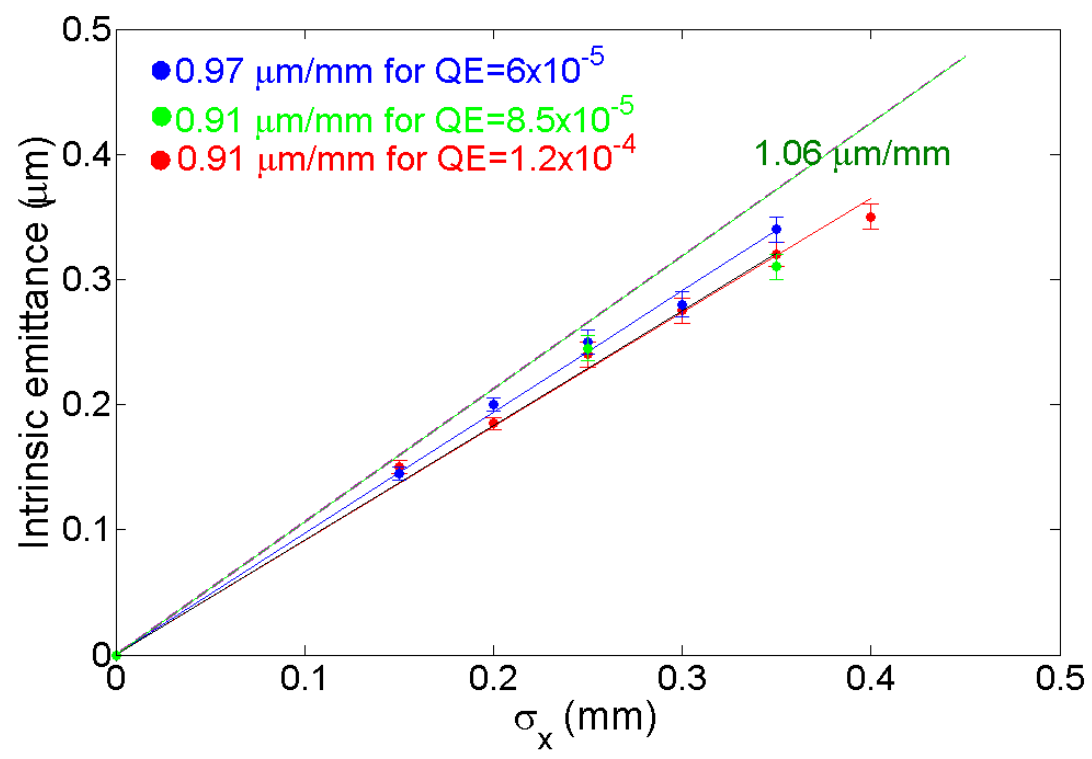

Fig.10. Measured intrinsic emittance with different QE following the laser cleaning at the LCLS. $\sigma_{x}$ is the rms laser spot size on the cathode. 


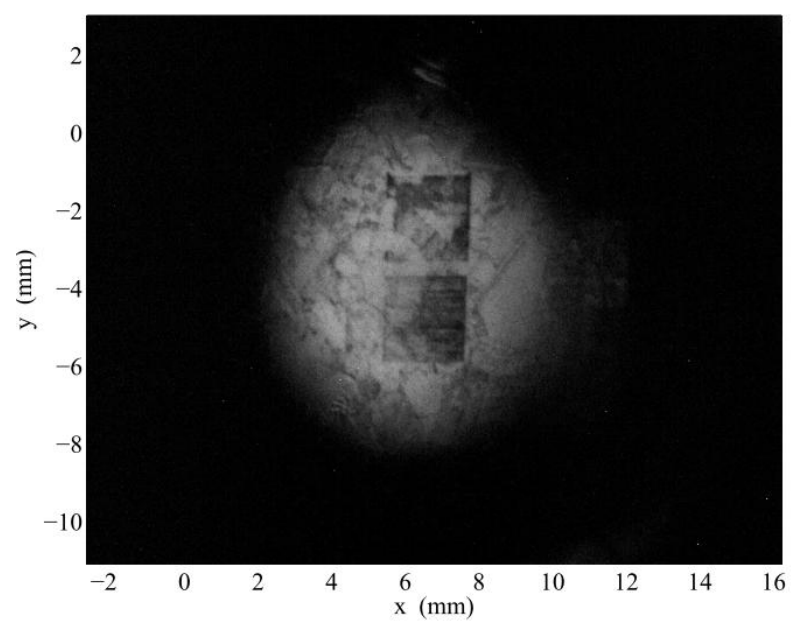

Fig.11. Image of the copper cathode using white light. The two square areas were processed by laser cleaning.

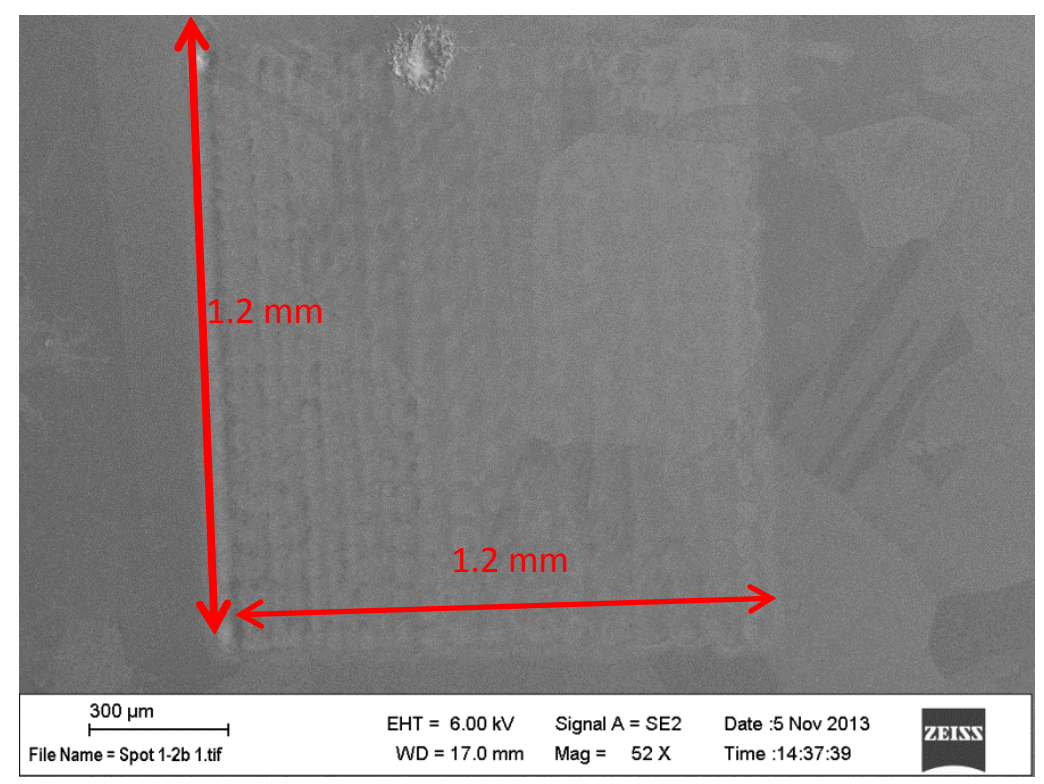

Fig. 12. Example of SEM image for the laser cleaned area $(1.2 \mathrm{~mm} \times 1.2 \mathrm{~mm})$. The small dips are caused by laser cleaning with $30 \mu \mathrm{m}$ rms of laser beam rastering through the cathode surface.

\section{Summary}

Historically LCLS cathodes have been exposed to air prior to installation in the RF gun due to the lack of a loadlock system, like most metal cathode RF gun systems in many other labs. Experimental studies demonstrate that the plasma cleaning for copper cathodes in the test chamber followed by the air exposure for installation in the gun results in a very low initial QE in the LCLS RF gun. All four ASTA cathodes, without the plasma cleaning in the test chamber, have reliably achieved initial $\mathrm{QE}>4 \times 10^{-5}$ in the ASTA gun with the cathode of $\mathrm{QE}<1 \times 10^{-7}$ in the test chamber prior to the installation in the gun. The high power RF processing with Schottky enhancement contributed to the $\mathrm{QE}$ boost by two orders of magnitudes. The in-situ cleaning for the cathode in the RF gun is essential to maintain good QE during the operations. The Laser cleaning technique for the LCLS-style copper cathode was significantly advanced at ASTA, resulting in a much better QE and emittance evolution than the previous cleaning 
procedure at the LCLS. The Physics of the laser cleaning process at the copper cathode surface was investigated. It is believed that the work function is not the major factor for the QE boost during the laser cleaning process. The change of cathode surface reflectivity is considered to be one of the main factors for the QE enhancement due to laser cleaning. A more complete picture of the complex laser-cleaning process is under investigation.

\section{Acknowledgements}

This work is within the framework of LCLS FEL R\&D program, supported by DOE under contract No. DEAC02-76SF00515. The authors would like to thank J. Eichner, J. Lewandowski, and S. Vetter for the assistances.

\section{References}

[1] F. Zhou et al., "High-brightness Electron Beam Evolution following Laser-based Cleaning of a Photocathode", Phys. Rev. ST Accel. Beams 15, 090703 (2012).

[2] E. Jongewaard et al., "RF Gun Photocathode Research at SLAC", in Proc. of 3rd International Part. Accel. Conference, New Orleans, Louisiana, 2012, pp. 664-666.

[3] R. Akre et al., "Commissioning the Linac Coherent Light Source Injector", Phys. Rev. ST Accel. Beams 11, 030703 (2008).

[4] A. Brachmann et al., "LCLS RF Gun Cathode Performance", in Proc. of 2nd International Part. Accel. Conference, San Sebastian, Spain, 2011, pp. 3200-3202.

[5] T. Srinivasan-Rao, I. Ben-Zvi, J. Smedley, X. J. Wang, M. Woodle, and D. T. Palmer, in Proc. of the Particle Accelerator Conference, Vancouver, BC, Canada, 1997 (IEEE, New York, 1997).

[6] X. J. Wang, T. Shrinivasan-Rao, K. Batchelor, I. Ben-Zvi, and J. Fischer, Nucl. Instr. Methods, Section A 356, 159 (1995).

[7] Dowell et al., "In situ cleaning of metal cathodes using a hydrogen ion beam", PRSTAB 9, 063502 (2006).

[8] D. Dowell and J. Schmerge, "Quantum Efficiency and Thermal Emittance of Metal Photocathodes", Phys. Rev. ST Accel. Beams 12, 119901 (2009). 
Reply: we think the referee's comment is correct. We remove the sentence and another sentence before the sentence related to previous fig 8 . We also think previous fig8 is not necessary so we removed it as well.

6- The last sentence of 3.2 states "With the standard gentle laser cleaning, no dark current increase is observed at ASTA.". A sentence describing if the "non-gentle" cleaning increases dark current should be added.

Reply: it is added at the last paragraph in section 3.2 .

7- At the beginning of section 3.4, it is stated that "In the past, laser cleaning had been considered as a chemical process similar to hydrogen cleaning.". This is not clear and should be explained in more detail, and/or a proper reference should be used.

Reply: we added/modified couple sentences in the 1st paragraph of section 3.4 .

8- Add omega and Qw in the list of definitions in section 3.4.

Reply: added.

9- In section 3.4, it is described that a change in reflectivity is observed in the areas where the laser cleaning was performed. This is a central point of the physics explanation of the results. Were quantitative reflectivity measurements performed to verify that the change is consistent with the increased QE? The addition of this information would strongly improve the value of the manuscript.

Reply: we did not measure the reflectivity of the laser cleaned area. But we did measure the reflectivity before and after RF processing for a non-laser-cleaned area. Before RF processing, the reflectivity is $30 \%$ and after the RF processing is only $10 \%$. And the QE is found increased after the RF processing. We added one sentence for the reflectivity measurements at the end of the 1st paragraph of section 2.2.

10- Always in section 3.4, a SEM measurement of the cathode surface is mentioned. A figure with the SEM image and some description of it should be added.

Reply: added. Fig12.

11-Last, an English revision of the whole manuscript would significantly improve the readability of the manuscript.

Reply: we re-smoothed the English. 